\title{
DISCUSSION (Continued)
}

B. G. Marsden: If it were as large as that on comet Arend-Roland, I certainly think so. I don't know why some of the long-period comets show nongravitational forces and others don't.

F. L. Whipple: There is always the worry that the optical center of light that you measure may not reflect the center of the actual mass, namely, the nucleus, I don't know how to resolve that problem. It could upset these calculations on the single-apparition comets.

B. G. Marsden: I agree, al though in some cases there are residuals of up to five or six seconds of arc, and for the long-focus observations I think it unlikely that the difference between center of light and center of mass could be so large. However, if the results from single-apparition comets were all the evidence we had on the existence of nongravitational forces on comets, I should be very skeptical

D. A. Mendis: I would like to ask a question about the effect of the evaporating gases on the spin of a comet if the comet is not very regular In that case, the axis of the expanding gas need not necessarily pass through the center of mass of the comet and this would give it a kick, not only in linear momentum but also in angular momentum, and this could probably either spin up or spin down the comet.

F. L. Whipple: Yes. I have spent quite a bit of time worrying about why comets split, particularly in the case of the new comets. The most rational explanation seems to be that they spin up.

It is almost impossible to find any other solution that will cause a comet to split except asymetric ejection of material that will cause a spin up. And you can very easily postulate shapes and conditions under which that effect would be very marked.

So it is quite possible, but how do you prove it?

W. Jackson: I thought Opik once wrote a paper saying that splitting of comets could occur through gravitational - breakup inside the Roche limit of the sun, I think is the word.

F. L. Whipple: That of course is in the sun-grazing comet families-the sun-grazing comets. There you do split off pieces. But the fact that the nucleus remains has been one of the strongest arguments for a discrete nucleus because if you had a gravel bank, nothing would remain. 